Grundriß der Rhetorik 
Gert Ueding/Bernd Steinbrink

\section{Grundriß \\ der Rhetorik}

Geschichte - Technik - Methode

3., überarbeitete und erweiterte Auflage

Verlag J.B. Metzler

Stuttgart - Weimar 
CIP-Kurztitelaufnahme der Deutschen Bibliothek

\section{Ueding, Gert:}

Grundriss der Rhetorik : Geschichte, Technik, Methode / Gert

Ueding; Bernd Steinbrink. - 3., überarb. und erw. Aufl. -

Stuttgart ; Weimar : Metzler, 1994

ISBN 978-3-476-01236-4

NE: Steinbrink, Bernd:

Dieses Werk einschließlich aller seiner Teile ist urheberrechrlich geschützr. Jede Verwertung außerhalb der engen Grenzen des Urheberrechtsgesetzes ist ohne Zustimmung des Verlages unzulässig und strafbar. Das gilt insbesondere für Vervielfältigungen, Übersetzungen, Mikroverfilmungen und die Einspeicherung und Verarbeitung in elektronischen Systemen.

ISBN 978-3-476-01236-4

ISBN 978-3-476-03195-2 (eBook)

DOI $10.1007 / 978-3-476-03195-2$

(C) 1994 Springer-Verlag GmbH Deutschland

Ursprünglich erschienen bei J.B. Metzlersche Verlagsbuchhandlung

und Carl Ernst Poeschel Verlag GmbH in Stuttgart 1994

EIN VERLAG DER 
Der wahre Redner ist, im Sinne Quintilians, in erster Linie immer Pädagoge: Aus Gründen der Erziehung, und nicht um des sophistischen Blendungsspiels willen, hat Lessing so sehr die wichtigen Aporien geschätzt - sie dienten seinem Appell, das Gegebene nicht als Fatalität aufzufassen, sondern immer auch das Gegenteil zu bedenken, sich auszumalen, die Welt wäre anders als jetzt, das Potentielle sei praktikabel und die Dinge stünden plötzlich auf dem Kopf. Walter Jens 


\section{Inhaltsverzeichnis}

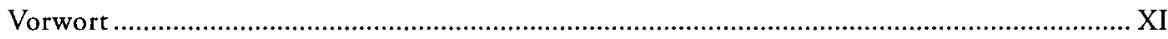

Vorwort zur dritten Auflage ..................................................................................... XII

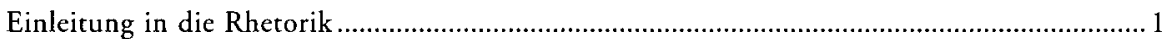

\section{Historischer Teil:}

A. Die Begründung der Rhetorik in der Antike ................................................ 11

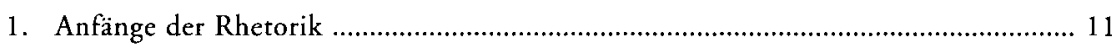

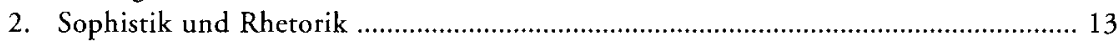

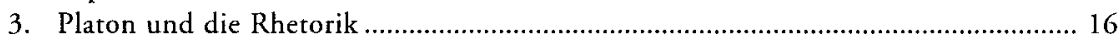

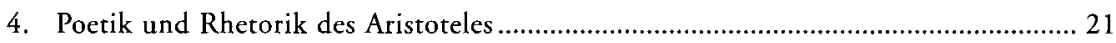

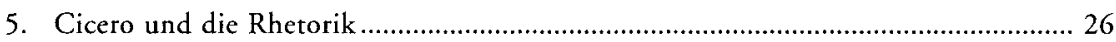

6. Kritik am Verfall der Beredsamkeit / Pseudo-Longinos ............................................. 37

7. Quintilian und die Ausbildung zum Redner ............................................................. 40

B. Christliche Erbschaft der Rhetorik im Mittelalter .............................................46

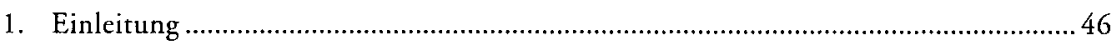

2. Augustinus und die christliche Beredsamkeit ........................................................... 48

3. Die artes liberales im Mittelalter .............................................................................. 53

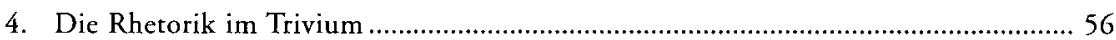

5. Die Autoritäten des mittelalterlichen Rhetorikunterrichts ...........................................61 61

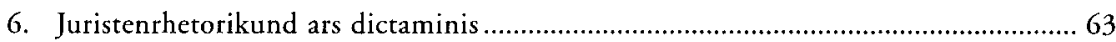

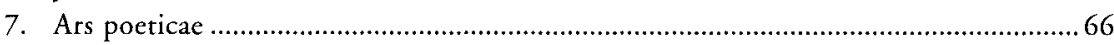

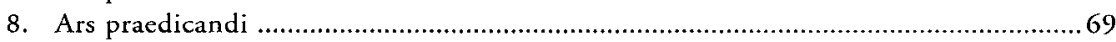

C. Studia humanitatis und Barockstil - Die Rhetorik vom 15. bis zum

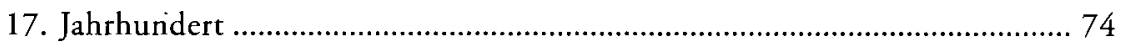

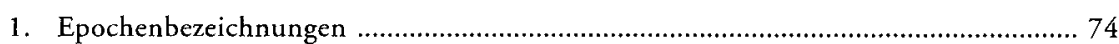

2. Die Wiederentdeckung und das Studium der alten Schriftsteller ..............................74

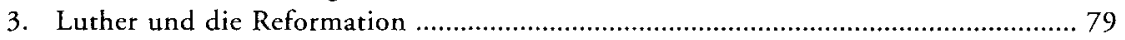

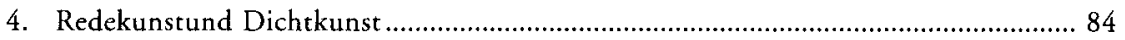

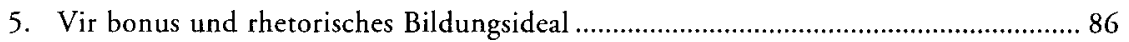

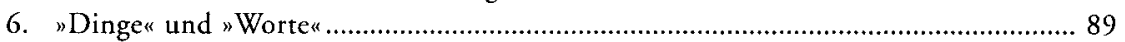

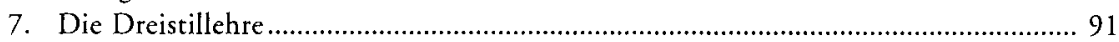

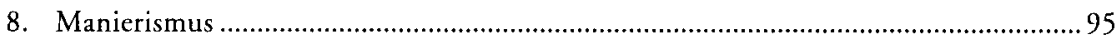

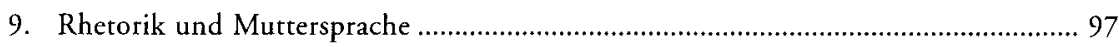

D. Rhetorik der Aufklärung - Das 18. Jahrhundert in Deutschland .................. 100

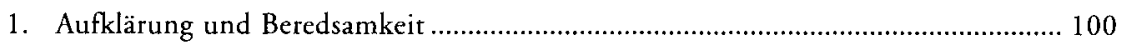

2. Begriff und Zweck aufklärerischer Redekunst ....................................................... 101

3. Die Bearbeitungsphasen der Rede ……......................................................... 108 


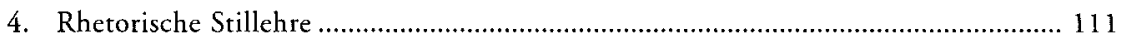

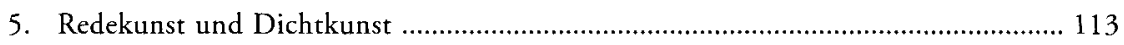

6. Rednerideal und bürgerliche Erziehung von Thomasius bis Knigge ....................... 115

7. Die Beredsamkeit nach ihren wichtigsten Gattungen ............................................. 122

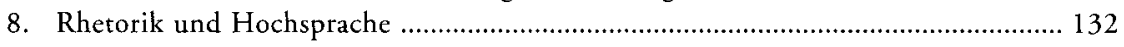

E. Ubiquität der Rhetorik. Vom Verfall und Weiterleben der Beredsamkeit

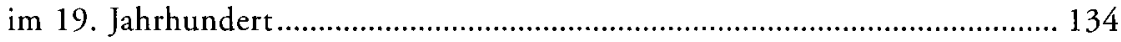

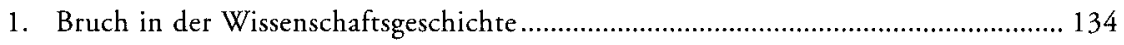

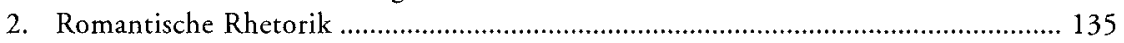

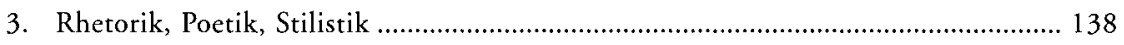

4. Literaturkritik und Literaturgeschichtsschreibung .................................................. 140

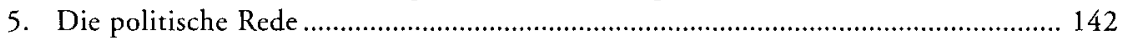

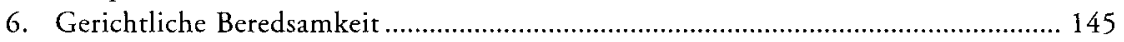

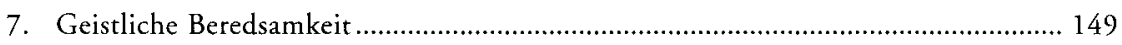

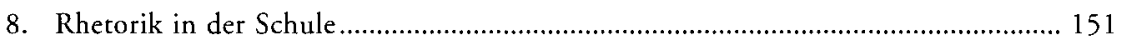

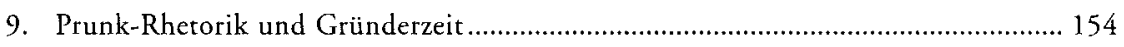

F. Aspekte moderner Rhetorik-Rezeption - Das 20.Jahrhundert ...................... 157

1. Rhetorik-Renaissance und apokryphe Rezeption ................................................. 157

2. Literaturwissenschaft und Literaturkritik ............................................................. 158

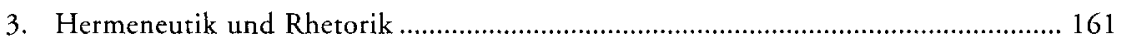

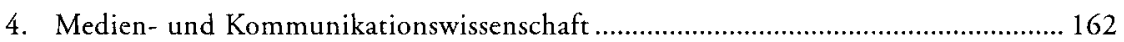

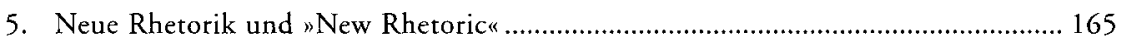

6. Philosophie und Rhetorik .......................................................................... 171

7. Die politische Beredsamkeit ................................................................................... 178

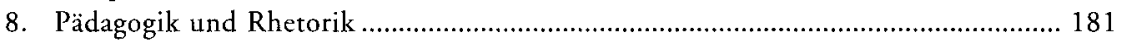

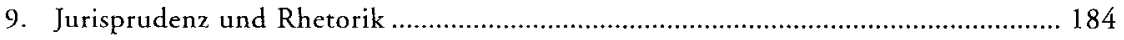

10. Predigtlehre .................................................................................................. 187

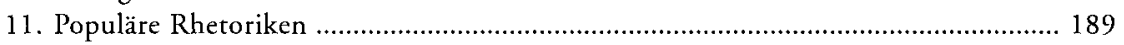

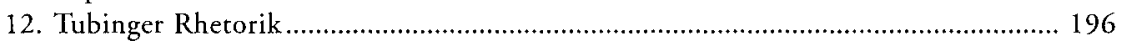

\section{Systematischer Teil:}

A. Die Produktionsstadien der Rede (erga tou rhetoros / opera oratoris partes artis) ..... 209

I. Das Finden und Erfinden des Stoffes (heuresis / inventio) ................................... 209

II. Die Ordnung des Stoffes (taxis / dispositio) ........................................................... 210

1. Das natürliche Ordnungsprinzip (ordo naturalis) .......................................... 211

2. Das künstliche Ordnungsprinzip (ordo artificialis) ....................................... 212

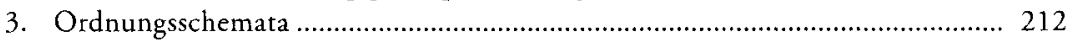

a) Die zweigliedrige, antithetische Disposition .......................................... 212

b) Die dreigliedrige Disposition ................................................................. 212

c) Die viergliedrige Disposition ................................................................ 212

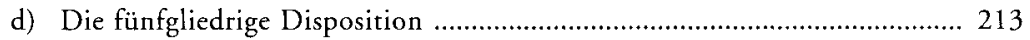

e) Die mehrgliedrige Disposition ................................................................. 213

III. Der sprachliche Ausdruck (lexis, hermeneia / elocutio) ........................................ 213 
1. Angemessenheit (prepon / aptum, decorum) ........................................... 216

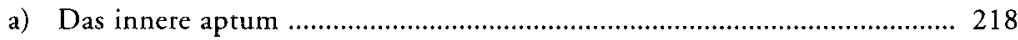

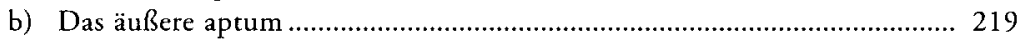

2. Sprachrichtigkeit (hellenismos / latinitas, puritas) ....................................... 221

a) Sprachrichtigkeit bei Einzelwörtern (latinitas in verbis singulis) .............. 222

b) Sprachrichtigkeit in Wortverbindungen (latinitas in verbis coniunctis) ..... 222

3. Deutlichkeit (sapheneia / perspicuitas) ....................................................... 224

a) Die Deutlichkeit der Einzelwörter (perspicuitas in verbis singulis) ............ 225

b) Die Deutlichkeit in Wortverbindungen (perspicuitas in verbis coniunctis). 225

4. Stufenfolge der Rede- und Schreibweisen (charakteres tes lexeos /

genera dicendi, genera elocutionis) .......................................................... 226

a) Die schlichte Stilart (charakter ischnos / genus subtile, genus bumile) ........ 227

b) Die mittlere Stilart (charakter mesos, charakter miktos / genus medium, genus mixtum).

c) Die großartige, pathetisch-erhabene Stilart (charakter megaloprepes, charakter hypselos / genus grande, genus sublime) ................................ 228

IV. Das Einprägen der Rede ins Gedächtnis (mneme / memoria) .............................. 229

V. Vortrag und Körperliche Beredsamkeit (hypokrisis / pronuntiatio, actio) .............. 230

B. Die Beweise und ihre Fundstätten (pisteis / probationes) ................................. 233

I. Einteilung der Beweise ......................................................................... 234

1. Natürliche Beweise (pisteis atechnoi / probationes inartificiales) ..................... 234

2. Kunstgemäße Beweise (pisteis entechnoi / probationes artificiales) .................. 234

II. Fundstätten der Beweise (topoi / loci) ........................................................ 234

1. Die sich aus der Person ergebenden Fundorte (loci a persona) ....................... 238

2. Die sich aus dem Sachverhalt ergebenden Fundorte (loci a re) ...................... 244

C. Redegegenstände und Redegattungen ........................................................... 254

I. Redegegenstände (materiae) ...................................................................... 254

II. Erkennen des Redegegenstandes (intellectio) .................................................. 254

III. Gliederung der Redegegenstände ........................................................................ 255

1. Die Gliederung der Redegegenstände nach den Fragen (quaestiones) ............... 255

2. Die Gliederung der Redegegenstände nach dem Verhältnis Redegegenstand/Zuhörer .......................................................................... 256

3. Die Gliederung der Redegegenstände nach dem Verhältnis Zuhörer/Redegegenstand

D. Redeteile (mere tou logou / partes orationis) ............................................ 258

I. Die Einleitung (prooimion / exordium) ..................................................................... 258

1. Die direkte Einleitung (principium) .......................................................... 259

a) Das Erlangen der Aufmerksamkeit (attentum parare) ............................... 259

b) Die Erweiterung der Aufnahmefähigkeit (docilem parare) ......................... 260

c) Das Erlangen des Wohlwollens (captatio benevolentiae) .......................... 260

2. Die indirekte Einleitung (insinuatio) ........................................................ 260

II. Die Erzählung (diegesis / narratio) ..................................................................... 261

1. Die Tugenden der Erzählung (aretai tes diegeseos / virtutes narrationis) ........... 261

2. Funktion und Gebrauch der Erzählung .................................................... 261

3. Die Darlegung des Themas (prothesis / propositio) ......................................... 262

4. Die Abschweifung (parekbasis / digressio) .................................................... 262

III. Die Beweisführung (pistis, eikos / argumentatio) ............................................ 263 
1. Gliederung als Eingang der Beweisführung (prothesis, prokataskeue / divisio, partitio). 264

2. Die Teile der Beweisführung ....................................................................... 264

3. Beweisarten (pisteis / probationes) ................................................................ 264

a) Beweisführung ohne Kunstmittel (pisteis atechnoi / probationes inartificiales) ...... 265

b) Beweisführung durch Kunstfertigkeit (pisteis entechnoi / probationes artificiales) .......................................................................... 265

b1) Zeichen, Indizien (semeion, tekmerion / signa) .................................. 266

b2) Die Beweisgründe (syllogismoi, enthymemata / ratiocinatio, argumenta) .. 266

b3) Das Beispiel (paradeigma / exemplum) ................................................ 267

b4) Die Sentenz (gnome / sententia) ...................................................... 268

c) Die Vergrößerung oder Steigerung (auxesis / amplificatio) ........................ 271

IV. Der Redeschluß (epilogos / peroratio) .................................................................... 274

1. Zusammenfassende Aufzählung (enumeratio) ................................................... 274

2. Affekterregung (affectus) ........................................................................ 274

E. Die Wirkungsfunktionen der Rede (officia oratoris) ........................................... 277

I. Einsicht und Belehrung (pragma / docere, probare) ............................................. 279

II. Unterhalten und Vergnügen (ethos / delectare, conciliare) ..................................... 280

III. Leidenschaftserregung (pathos / movere, concitare) ............................................... 280

F. Der Redeschmuck (kosmos / ornatus) .......................................................... 283

I. Allgemeine Mittel der Rede zur Steigerung des Ausdrucks ................................. 284

II. Der Redeschmuck in den Einzelwörtern (ornatus in verbis singulis) ...................... 286

1. Archaismus (antiquitas) ........................................................................... 286

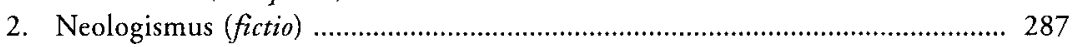

3. Tropus (tropos / verbum translatum) ........................................................... 287

III. Der Redeschmuck in Wortverbindungen (ornatus in verbis coniunctis) ................. 299

1. Die Wortfiguren (schemata lexeos / figurae verborum) ..................................... 301

a) Durch Hinzufügung (per adiectionem) gebildete Wortfiguren ................... 302

b) Durch Auslassung (per detractionem) gebildete Wortfiguren ...................... 305

c) Durch Umstellung (per transmutationem) gebildete Wortfiguren .............. 306

2. Die Gedankenfiguren, Sinnfiguren (schemata dianoias, figurae sententiae) ...... 308

a) Durch Veränderung der Satzordnung oder Satzart gebildete Gedankenfiguren ....................................................................... 310

b) Durch Sinnpräzisierung oder Sinnaussparung gebildete Gedankenfiguren .... 313

c) Durch szenische Erweiterung der Rede und Publikumsansprache gebildete Gedankenfiguren ....................................................................... 319

3. Die Wortfügung (synthesis / compositio) ……………………........................... 323

G. Die Übung (askesis, melete / exercitatio, usus) ........................................ 328

I. Lese- und Hörübungen (legendo, audiendo) .................................................... 329

II. Schreibübungen (scribendo) …….................................................................... 330

III. Redeübungen (dicendo) ............................................................................. 331

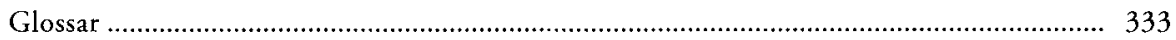

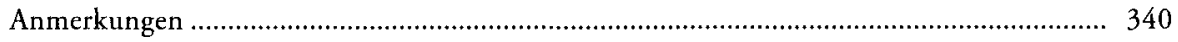

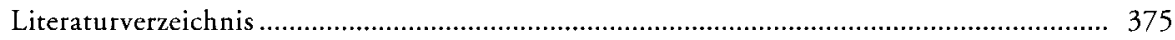

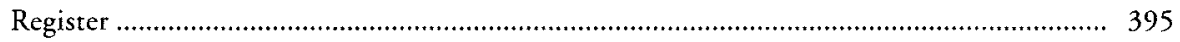




\section{Vorwort zur zweiten Auflage}

Als 1976 die von mir zusammen mit den Teilnehmern eines Oberseminars erarbeitete und herausgegebene "Einführung in die Rhetorik - Geschichte, Technik, Methode erschien, waren den Autoren die Schwächen des Buches wohl am deutlichsten bewußt. Doch stimmten alle Beteiligten, auch im Verlag und unter den Kollegen, die um Rat gefragt wurden, darin überein, daß der dringende Mangel an einem derartigen rhetorikgeschichtlichen Lese- und Arbeitsbuch unstreitig das größere Übel sei gegenüber den Lücken und Uneinheitlichkeiten des damals vorliegenden Manuskripts, das in dem einmal gesetzten personellen, zeitlichen und konzeptionellen Rahmen nur unwesentlich zu verändern gewesen wäre. Der Erfolg des Buches hat uns, soweit wir das überblicken können, recht gegeben, und fast ausnahmslos stimmten indirekt auch seine Rezensenten durch ihr Urteil dieser Entscheidung zu. Es sind teilweise außerordentlich umfangreiche, gründliche und hilfreiche Besprechungen erschienen (Scheerer, Kopperschmidt, Plett, Rohner, Ockel, um nur einige zu nennen), deren Einwände und Hinweise bei der völligen Neubearbeitung des Buches dankbar benutzt wurden. Kaum ein Satz ist auf dem anderen geblieben, allein das Kapitel "Antike Rhetorik", schon für die Auflage 1976 von Bernd Steinbrink bearbeitet, konnte erhalten bleiben und wurde nur etwas ergänzt und vervollständigt. Alle anderen Kapitel der Rhetorikgeschichte vom Mittelalter bis zur Gegenwart wurden von Bernd Steinbrink und mir (unter gelegentlicher Berücksichtigung der ursprünglichen Beiträge natürlich) vollständig neu geschrieben. Die Darstellung ist so kohärent, materialreicher und lückenloser geworden, hoffentlich dadurch auch lesbarer und überzeugender. Freilich ist das Buch damit auch aus dem Einführungs-Stand herausgewachsen, was beim Vergleich mit anderen, inzwischen cinschlägigen Publikationen sichtbar wird, die sich auf knappstem Raum wirklich mit einführenden Abrissen und Hinweisen begnügen.

Dennoch bleiben Wünsche offen, der Kenner und Spezialist wird manches vermissen, anderes unzureichend erörtert finden - das ist bei Büchern dieser Art (wie bei aller Geschichtsschreibung) wohl unvermeidlich. Ein ausdrückliches Wort aber noch zum zweiten (systematischen) Teil. Auch er wurde überarbeitet, ergänzt und dadurch hoffentlich verbessert, doch ein prinzipieller Mangel ließ sich nicht beheben, den einige Rezensenten zutreffend als Widerspruch von geschichtlicher Perspektive (des ersten Teils) und unhistorischer Systematik namhaft gemacht haben, weil darin eine rhetorische communis opinio unterstellt werde (noch mit dem Anschein wissenschaftlicher Systematik), die zuvor doch gerade in ihre EntwicklungsStadien aufgelöst wurde.

Kein Zweifel: eine kategorien- und begriffsgeschichtliche Herleitung der Rhetorik auch hinsichtlich ihres Systems wäre vom Standpunkt historischer Forschung, der wir vor anderen Methoden den Vorzug geben, die angemessene Fortführung, sie kann aber weder im Rahmen einer Einführung noch eines Grundrisses geleistet werden. Doch sei schon hier darauf hingewiesen, daß seit Mai 1985 ein am Seminar für Allgemeine Rhetorik der Universität Tübingen eingerichtetes Forschungsprojekt die 
Aufgabe verfolgt, ein "Historisches Sachwörterbuch der Rhetorik" zu erarbeiten. Doch auch dies wird systematische Querschnitte in der hier vorgelegten Art nicht überflüssig machen, die man als pragmatische Instrumente betrachten sollte, die solange und soweit gerechtfertigt sind, wie sie ihren Dienst tun, also brauchbar sind und zur Erkenntnis menschlicher Rede, ihrer Machart und Wirkungsweise ebenso taugen, wie sie die eigene Rede- oder Textproduktion des Lesers zu befördern vermögen.

Tübingen 1985

Gert Ueding

\section{Vorwort zur dritten Auflage}

Lehrbuch-Autoren und Philosophen haben mindestens eines gemeinsam: sie bessern ein Leben lang an ihren Gebäuden, und so hoffe ich, daß diese dritte neubearbeitete Auflage des "Grundrisses der Rhetorik« mit ihren Kommentaren, Ergänzungen und Korrekturen dem praktischen Nutzen noch besser gerecht wird als die beiden Vorgänger. Zu danken ist auf jeden Fall allen kritischen Lesern, die mich auf Irrtümer oder Ungenauigkeiten aufmerksam gemacht haben, allen voran Alwin Binder in Münster, dessen scharfem Blick kein Widerspruch, keine Flüchtigkeit entgangen ist.

Auch diesmal muß ich mich wieder bei meinen Mitarbeitern am Seminar für Allgemeine Rhetorik der Universität Tübingen (und insbesondere bei Boris Kositzke und Heinzpeter Lindroth) bedanken, die mir bei Revision und Fahnenkorrektur behilflich waren, geduldig Zitate verifiziert und Forschungsliteratur gesichtet haben. Verbessert und - in Zitaten und Beispielen - vereinheitlicht wurde der systematische Teil, wesentlich ergänzt das Kapitel über die praktische Rhetorik der Gegenwart, völlig neu gefaßt und dem erreichten Stand angepaßt das Kapitel über die Tübinger Rhetorik und schließlich hinzugefügt der Teil über Philosophie und Rhetorik; auch das Register wurde vollkommen neu eingerichtet und die Lücken in der Bibliographie wurden gefüllt.

"Lesen heißt borgen, daraus erfinden, abtragen« meinte Lichtenberg, und es gibt keine bessere Maxime für den Umgang mit einem Lehrbuch als diese.

Tübingen 1994

Gert Ueding 